\title{
¿Cuáles son los Rangos de Normalidad de los Diámetros de la Aorta Infrarrenal, Medidos con Ultrasonografía 2D, en Sujetos sin Antecedentes de Enfermedad Vascular?
}

\author{
What are the Normal Ranges of the Infrarenal Aortic Diameters Measured \\ with 2D Ultrasound in Subjects with no History of Vascular Disease?
}

\author{
Marcelo Lagos* \& Carlos Manterola*,****
}

LAGOS, M. \& MANTEROLA, C. ¿Cuáles con los rangos de normalidad de los diámetros de la aorta infrarrenal, medidos con ultrasonografía 2D, en sujetos sin antecedentes de enfermedad vascular? Int. J. Morphol., 34(3):1017-1023, 2016.

RESUMEN: El diámetro de la parte abdominal de la aorta (AAI), constituye un factor relevante para decidir reparar un aneurisma aórtico abdominal. El objetivo de este estudio es determinar rangos de normalidad de los diámetros de laAAI, medidos con ultrasonografía 2D, en sujetos sin antecedentes de enfermedad vascular. Estudio de corte transversal, realizado en los Hospitales Regional de Temuco y de Pitrufquen. Se estudiaron 399 sujetos mayores de 15 años, sin antecedentes de enfermedad vascular, que consultaron por cuadros de dolor abdominal. Se realizó ultrasonografía 2D con dos equipos diferentes (General Electric RT3200 Advantage II y Medison co. Ltda. Modelo SA-600; ambos con transductores de 3,5 Mhz.) y por dos observadores independientes. Las variables resultado fueron diámetros antero-posterior (DAP) y transverso (DT) de laAA. Otras variables de interés fueron: sexo, edad índice peso/talla y superficie corporal. Se aplicó estadística descriptiva, inferencial aplicando t test para variables continuas, $\mathrm{x}^{2}$ para variables categóricas, ANOVA y Kruskal Wallis para comparación de grupos; estudios de correlación y una regresión lineal simple. El DAP promedio fue de 16,1 $\pm 2,2 \mathrm{~mm}$ (9 a $23 \mathrm{~mm}$ ) y el DT promedio fue de 19,4 $\pm 2,7 \mathrm{~mm}(11 \mathrm{a}$ $26 \mathrm{~mm})$. Se observaron DAP y DT significativamente mayores en el subgrupo de >50 años y en hombres. Hubo correlación positiva entre índice peso/talla-DAP $(\mathrm{p}=0,0321)$ e índice peso/talla-DT ( $\mathrm{p}=0,0052)$, más relevante en el subgrupo femenino. Por otra parte, se demostró correlación positiva entre DAP y superficie corporal $(\mathrm{p}<0.0001)$ y DT y superficie corporal $(\mathrm{p}<0.0001)$. Sexo, edad y superficie corporal se asocian con DAP y DT más elevados. Al parecer la población estudiada posee AAI de menor diámetro promedio que lo descrito en la literatura internacional.

PALABRAS CLAVE: Aorta; Parte abdominal de la aorta; Diámetro de la parte abdominal de la aorta; Aneurisma de la parte abdominal de la aorta; Ultrasonografía; Estudio de corte transversal.

\section{INTRODUCCIÓN}

Debido al incremento de edad de la población general en nuestras sociedades, es que ha existido un aumento en el número de casos de sujetos portadores de enfermedades que tradicionalmente están asociadas a la ancianidad. Destaca de entre éstas la enfermedad aneurismática de la parte abdominal de la aorta infrarrenal, sobre la cual, se ha reportado una incidencia creciente de acuerdo a los grupos de edad estudiados: $6 \%$ en la sexta década de vida (Valdés et al., 2003a), $10 \%$ en la séptima y hasta $12 \%$ en la octava (Singh et al., 2001; Valdés et al., 2003b).
El diámetro de la parte abdominal de la aorta infrarrenal (AAI), constituye el factor aislado de mayor importancia para decidir reparar un aneurisma aórtico abdominal (AAA) (Valdés et al., 1997; Valdés et al., 2003a; Valdés et al., 2003b). Sin embargo existen demasiadas diferencias en la literatura al momento de determinar una definición precisa de AAA; debido a lo cual, se ha recomendado definir como AAA al aumento del 50\% o más del diámetro aórtico infrarrenal, esto comparado con el diámetro aórtico esperable para el individuo según la edad y sexo de este; y

\footnotetext{
* Clínica Alemana de Temuco, Temuco, Chile.

** Departamento de Cirugía y Centro de Estudios Morfológicos y Quirúrgicos (CEMyQ), Universidad de La Frontera, Temuco, Chile.

${ }^{*}$ Centro de investigaciones biomédicas, Universidad Autónoma de Chile, Chile.
} 
según otros factores como el tamaño corporal (Bengtsson et al., 1996; Peón). Ahora, la determinación de estos "parámetros de normalidad" han sido estudiados por múltiples grupos en el extranjero (Yucel et al., 1991; Länne et al., 1992; Liddington \& Heather, 1992; Singh et al., 1998; Allison et al., 2008; Beales et al., 2011; Bonnafy et al., 2013; Gürtelschmid et al., 2014); pero éstas no necesariamente pueden ser extrapolables a la realidad chilena. Al revisar la literatura nacional sólo se existe un reporte previo, a partir de necropsias del Instituto Médico Legal de Santiago (Poblete et al., 1988).

Este artículo fue escrito siguiendo la iniciativa MInCir para el reporte de estudios observacionales descriptivos (Manterola \& Astudillo, 2013).

El objetivo de este estudio es determinar rangos de normalidad de los diámetros de la AAI, medidos con ultrasonografía $2 \mathrm{D}$, en sujetos sin antecedentes de enfermedad vascular.

\section{MATERIAL Y MÉTODO}

Diseño: Estudio de corte transversal.

Centro: El estudio fue realizado en los Servicios de Urgencia de los hospitales Regional de Temuco y de Pitrufquen.

Participantes: Se incluyeron 495 sujetos mayores de 15 años, sin antecedentes de enfermedad vascular, que consultaron de forma consecutiva en los dos centros antes señalados, por cuadros de dolor abdominal. Fueron excluidos aquellos casos en que se verificó diabetes mellitus, hipertensión arterial, tabaquismo y alcoholismo.

Muestreo: No probabilístico de casos consecutivos. La muestra, una vez aplicados los criterios de selección, quedó constituida por 399 sujetos.

Protocolo de medición: Se realizaron ecotomografías 2D en dos equipos de ultrasonido diferentes, en el Hospital Regional de Temuco se utilizó un equipo General Electric modelo RT3200 Advantage II con transductor de 3,5 Mhz.; y en el de Pitrufquén, un equipo marca Medison co. Ltda. Modelo SA-600, con transductor de 3,5 Mhz. Antes de iniciar las mediciones, se debió realizar una estandarización de éstas a través de un estudio piloto, para conocer la confiabilidad interobservador (Singh et al., 1998; Yucel et al., 1991), ya que serían realizadas por dos observadores independientes.
Variables: Las variables resultado fueron los diámetros anteroposterior (DAP) y transverso (DT) de la AAI, medida en $\mathrm{mm}$. Otras variables de interés fueron: sexo, edad (que se trabajó como variable categórica < 30 años, de 30 a 50 años y > 50 años), índice peso/talla aplicando la fórmula Peso $(\mathrm{kg}) /$ Talla $\left(\mathrm{cm}^{2}\right)$; y superficie corporal, aplicando la fórmula: $0,20247 \mathrm{x}$ estatura $(\mathrm{mt}) 0,725 \mathrm{x}$ peso $(\mathrm{kg}) 0,425$.

Seguimiento: No fue considerado por tratarse de un estudio transversal.

Estadísticas: Utilizando el paquete estadístico Stata 9.0, se realizó un análisis exploratorio de los datos. Se aplicó estadística descriptiva con cálculo de porcentajes, medidas de tendencia central, extrema, e intervalos de confianza del $95 \%$. Posteriormente se realizó estadística inferencial aplicando $\mathrm{t}$ test, para comparar variables continuas; $\mathrm{x}^{2} \mathrm{de}$ Pearson, para comparación de variables categóricas; y ANOVA y Kruskal Wallis para comparación de grupos. Además, se realizó un estudio de correlación entre las mediciones de los diámetros aórticos y algunas variables biodemográficas. Finalmente se realizó un análisis multivariado aplicando regresión lineal simple para estudiar el comportamiento de las variables biodemográficas respecto de las mediciones aórticas.

Principios éticos: Todos los pacientes firmaron su consentimiento informado y sus identidades se mantuvieron ocultas luego de que fueron codificados cada uno de ellos.

\section{RESULTADOS}

De la muestra de 399 pacientes, el $58 \%$ eran mujeres $(n=233)$ y la media de edad fue 40 años $(95 \%$ de la población estudiada tenía entre 38 y 42 años). La distribución por edad y sexo, se puede apreciar en las Tablas I y II.

Al analizar la distribución peso y estatura por sexo, se verificó que en los hombres los valores promedios fueron más altos en relación a las mujeres: $73,1 \mathrm{~kg}$ vs. $64,8 \mathrm{~kg}$; y $165,5 \mathrm{~cm}$ vs. $156,0 \mathrm{~cm}$ respectivamente ( $\mathrm{p}<0,0001$, en ambos casos).

Tabla I. Distribución de la muestra por sexo, según variables de interés. $(\mathrm{n}=399)$.

\begin{tabular}{lccc}
\hline & Hombres & Mujeres & $p$ \\
\hline Peso $(\mathrm{kg})$ & 73,1 & 64,8 & $<0,001$ \\
Talla $(\mathrm{cm})$ & 165,5 & 156,0 & $<0,001$ \\
Superficie Corporal $(\mathrm{mt} 2)$ & 1,8 & 1,6 & $<0,001$ \\
IMC $(\mathrm{kg} / \mathrm{mt} 2)$ & 26,6 & 26,7 & 0,95 \\
\hline
\end{tabular}


Tabla II. Distribución de la muestra por grupos de edad y sexo. $(n=399)$.

\begin{tabular}{lcccc}
\hline Edad & Mujeres & Hombres & \multicolumn{2}{c}{ Total } \\
\hline$<30$ años & 71 & 58 & 129 & $(32,3 \%)$ \\
$30-50$ años & 137 & 27 & 164 & $(41,1 \%)$ \\
$>50$ años & 25 & 81 & 106 & $(26,6 \%)$ \\
Total & 233 & 166 & 399 & $(100 \%)$ \\
\hline
\end{tabular}

$\mathrm{p}<0,00001$

Al estudiar la superficie corporal, se constató que ésta fue mayor en hombres que mujeres (1,80 $\mathrm{mt}^{2}$ vs. 1,63 $\mathrm{mt}^{2}$ respectivamente; $\left.\mathrm{p}<0,0001\right)$. Al comparar el índice peso/talla, se observaron valores promedios similares $\left(26,6 \mathrm{~kg} / \mathrm{mt}^{2}\right.$ en hombres vs. $26,7 \mathrm{~kg} / \mathrm{mt}^{2}$ en mujeres, $\mathrm{p}=0,9553)$. Al comparar estas variables por grupos de edad, se determinó que tanto el índice peso/talla como de la superficie corporal, fueron similares para los tres grupos, no existiendo diferencias estadísticamente significativas.

El DAP promedio de la AAI fue de 16,1 $\pm 2,2 \mathrm{~mm}$ (9 a $23 \mathrm{~mm}$ ) y el DT promedio fue de 19,4 $\pm 2,7 \mathrm{~mm}$ (11 a $26 \mathrm{~mm}$ ). Al desagrupar por sexo, se registraron diámetros de $17,9 \mathrm{~mm}$ y $14,9 \mathrm{~mm}$ para hombres y mujeres respectivamente.

Respecto de los DAP y DT de la AAI; se observaron diferencias estadísticamente significativas, dadas por diámetros mayores en el subgrupo de sujetos >50 años (Tabla III). Algo similar se verificó al estudiar estas mediciones según sexo, diámetros que resultaron mayores en los hombres (Tabla IV).

Al correlacionar DAP con edad, se determinó correlación positiva, pero con un grado de asociación bajo
Tabla III. Mediciones del DAP y DT de la AAI, por grupos de edad. $(\mathrm{N}=399)$.

\begin{tabular}{ccccc}
\hline Variables & $<\mathbf{3 0}$ años & $\mathbf{3 0 - 5 0}$ años & $>\mathbf{5 0}$ años & $\boldsymbol{p}$ \\
\hline DAP \pm DE $(\mathbf{m m})$ & $16,2 \pm 2,0$ & $15,2 \pm 2,6$ & $17,4 \pm 1,9$ & 0,0001 \\
Valor mínimo & 12 & 9 & 9 & \\
Valor máximo & 19 & 19 & 23 & \\
DT \pm DE $(\mathbf{m m})$ & $19,0 \pm 1,9$ & $18,3 \pm 3,1$ & $21,8 \pm 2,9$ & 0,0001 \\
Valor mínimo & 11 & 11 & 13 & \\
Valor máximo & 25 & 26 & 25 & \\
\hline
\end{tabular}

DAP: Diámetro anteroposterior. DT:Diámetro transverso. DE:Desviación estándar

Tabla IV. Valores de mediciones de los DAP y DT de la AAI según sexo. $(\mathrm{N}=399)$

\begin{tabular}{lccc}
\hline Sexo & DAP \pm DE $(\mathbf{m m})$ & DT \pm DE $(\mathbf{m m})$ & $\boldsymbol{p}$ \\
\hline Femenino & $14,9 \pm 2,3$ & $18,1 \pm 2,8$ & 0,0001 \\
Masculino & $17,9 \pm 1,3$ & $21,5 \pm 2,1$ & 0,0001 \\
TOTAL & $\mathbf{1 6 , 1} \pm \mathbf{2 , 4}$ & $\mathbf{1 9 , 5} \pm \mathbf{3 , 1}$ & $\mathbf{0 , 0 0 0 1}$ \\
\hline
\end{tabular}

DAP: Diámetro anteroposterior. DT:Diámetro. transverso. DE: Desviación estándar

(Fig.1). Al analizar las mediciones del DAP por sexo, no se determinó asociación ( $\mathrm{p}=0,443$ para mujeres y $\mathrm{p}=0,520$ para hombres).

Con el DT, se apreció correlación significativa tanto al analizar el total de la muestra (Fig. 2), como al desagrupar por sexo $(\mathrm{p}=0,0297$ y $\mathrm{p}=0,0000$ para mujeres y hombres respectivamente).

La correlación entre índice peso/talla y DAP fue positiva con el DAP ( $\mathrm{p}=0,0321$ ) (Fig. 3); pero al desagrupar por sexo se constató que el grupo masculino perdió significancia $(\mathrm{p}=0,8259)$, a diferencia del grupo femenino que aumentó $(\mathrm{p}=0,0075)$.

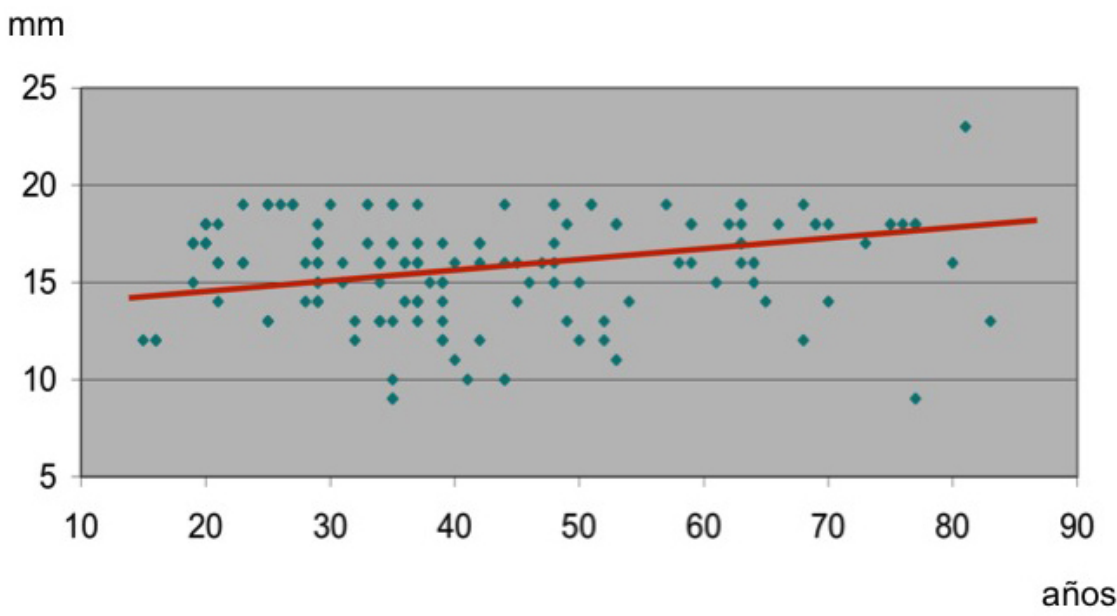

Fig. 1. Gráfico de correlación entre mediciones de DAP y edad. 

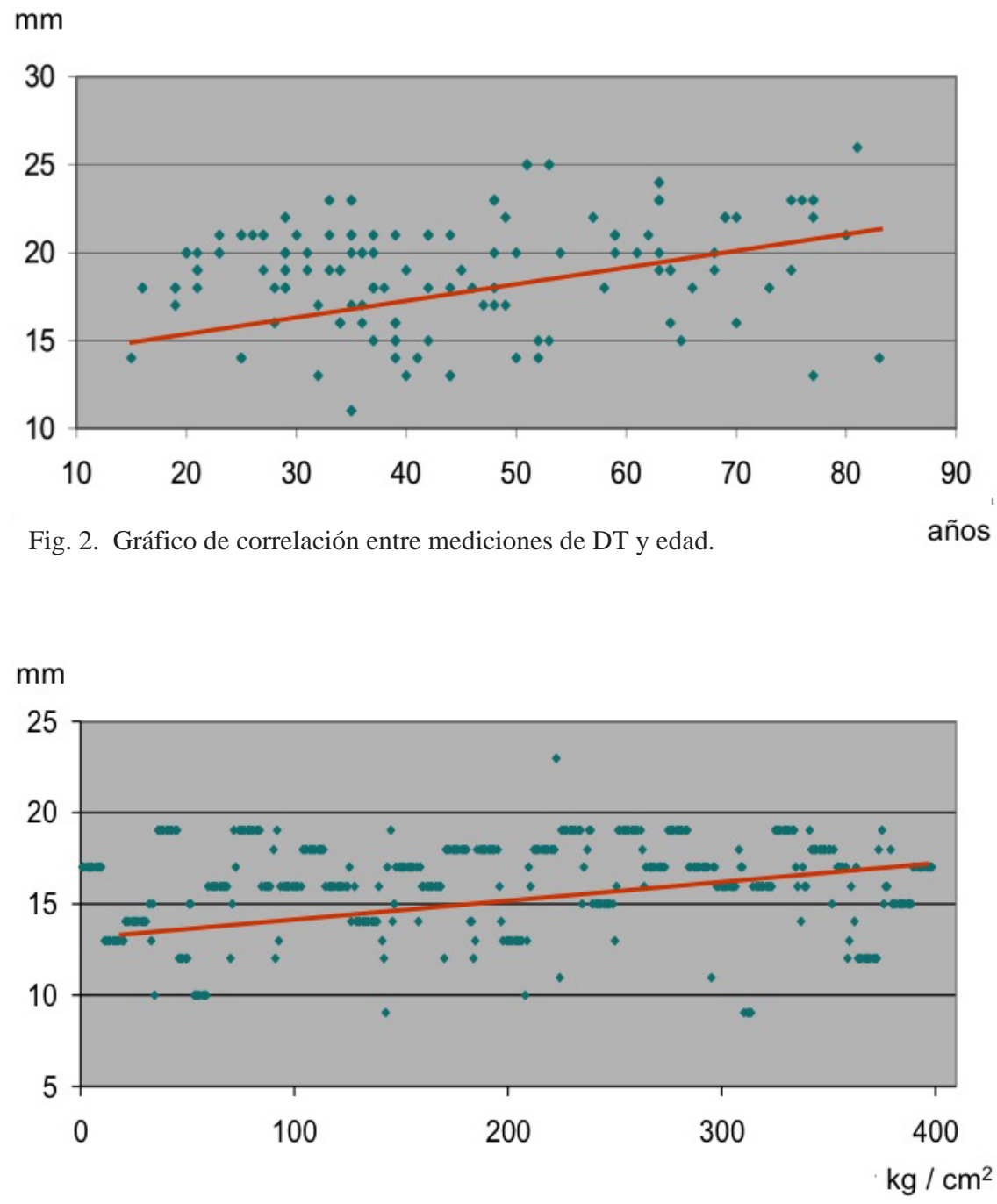

Fig. 3. Gráfico de correlación entre mediciones de DAP e índice peso/talla.

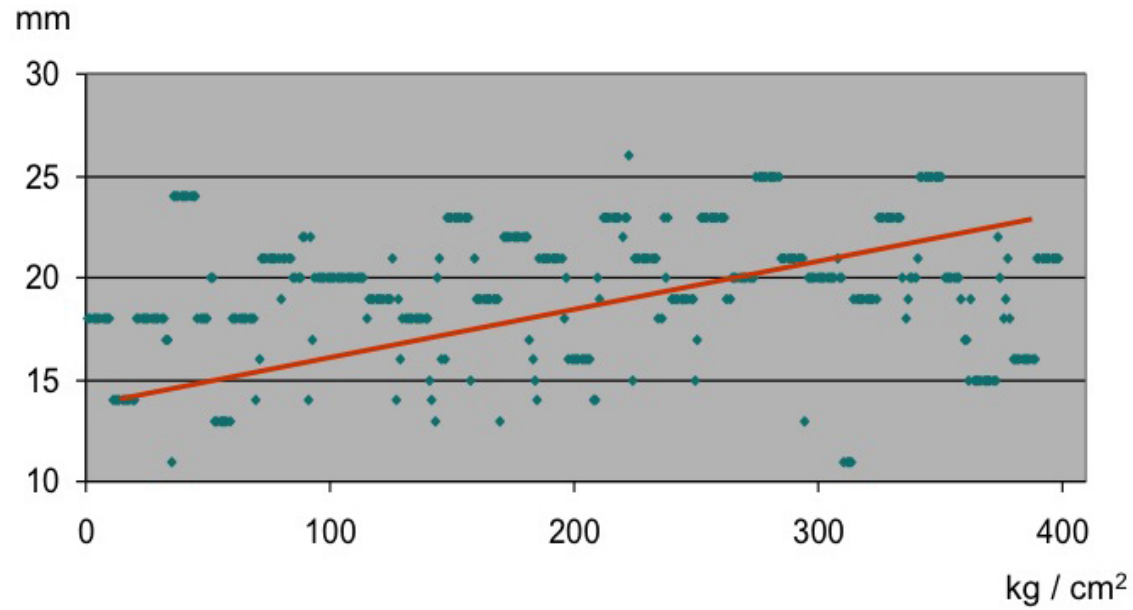

Fig. 4. Gráfico de correlación entre mediciones de DT e índice peso/talla. 


\section{$\mathrm{mm}$}

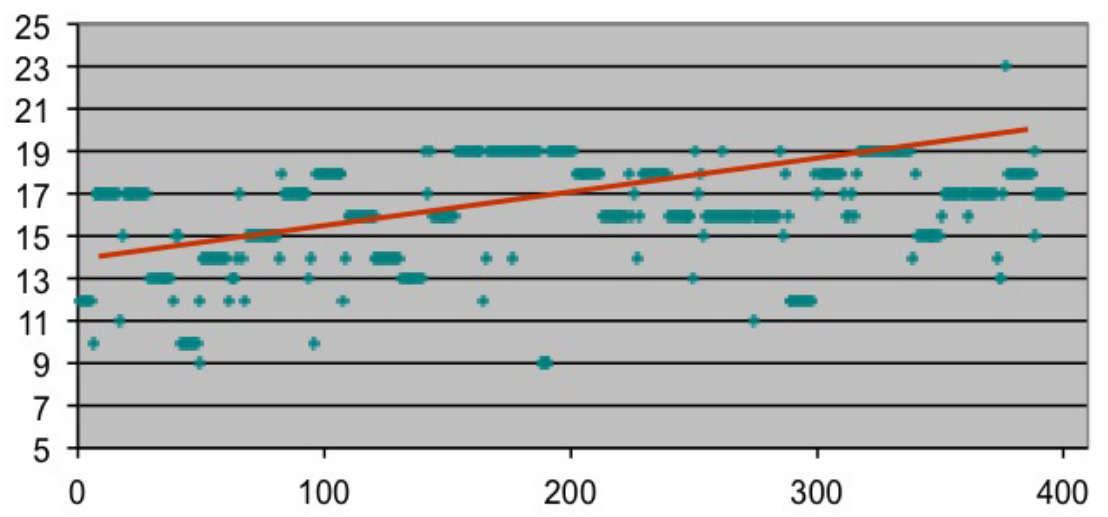

Fig. 5. Gráfico de correlación entre mediciones de DAP y superficie corporal.

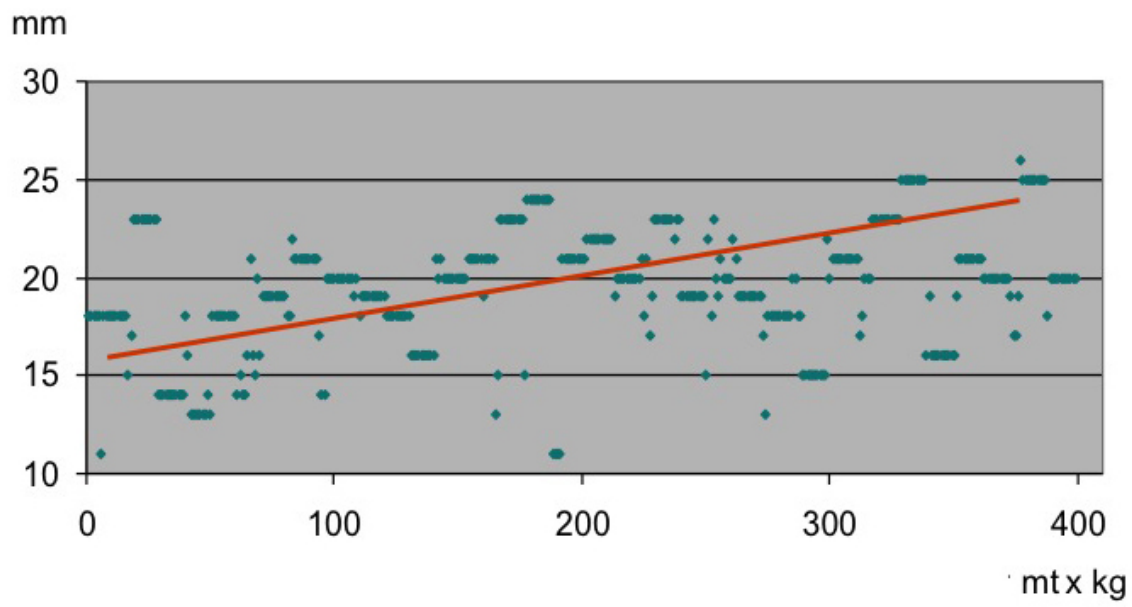

Con el DT se verificó algo similar, con correlación positiva para la muestra general $(\mathrm{p}=0,0052)$ (Fig. 4); y al desagrupar por sexos, el grupo de mujeres mantuvo significancia $(\mathrm{p}=0,0060)$ a diferencia del grupo de hombres que la perdió ( $\mathrm{p}=0,0797$ ).

Por otra parte, se demostró correlación positiva entre DAP y superficie corporal en la muestra total ( $<<0,0001$ ) (Fig. 5); lo que se mantuvo incluso al desagrupar por sexo ( $\mathrm{p}=0,0033$ en las mujeres y $\mathrm{p}=0,0495$ en los hombres). Y también correlación positiva entre DT y superficie corporal en la muestra total (p<0,0001) (Fig. 6); no así al desagrupar por sexo (masculino, $\mathrm{p}=0,1354$ y femenino $\mathrm{p}=0,3099$ ).

Fig. 6. Gráfico de correlación entre mediciones de DT y superficie corporal.

\section{DISCUSIÓN}

La relevancia del conocimiento del diámetro aórtico ha sido claramente demostrado, basta señalar que permite determinar el diagnóstico de AAA, lo que aún es controversial.

A nivel internacional se han desarrollado una serie de intentos tendientes a precisar la normalidad de valores de los DAP y DT de la AAI. La más relevante en este aspecto es un estudio cooperativo entre 15 centros médicos de veteranos de guerra de EE.UU., donde lograron reunir una muestra mayor de 69.000 individuos; y aunque se pueden discutir detalles metodológicos, sigue siendo patrón de comparación para cualquier nuevo estudio (Lederle et al., 1997). Los resultados de este estudio se orientaron exclusivamente a los DAP y DT; diámetro transverso del que existe evidencia que sugiere que sería menos confiable (Yucel et al., 1991); debido a que los límites laterales de la pared aórtica serían menos definidos, lo que podría inducir a errores en las mediciones; no obstante lo cual, en esta experiencia, se constataron hallazgos similares para ambos diámetros con esa serie. 
Por otra parte, el único estudio nacional encontrado en este sentido, se trató de una muestra de 117 hombres y 33 mujeres, de 12 a 91 años de edad. Los autores destacaron la variabilidad observadas tanto en la circunferencia de la AAI como de las arterias ilíacas comunes. Sin embargo objetivaron una correlación significativa entre edad y circunferencia aórtica $(\mathrm{p}=0,01)$, pero no entre esta última y la variable peso (Poblete et al., 1988).

Aunque existe evidencia de asociación entre ambos diámetros aórticos y la variable sexo (siendo el masculino el que presenta valores más altos), hecho constatado también en este estudio; el resto de las asociaciones que resultaron significativas podrían ser discutibles. Por ejemplo con la variable edad, en la encontramos que a mayor edad mayores eran los diámetros; sin embargo como se observa en la Tabla II, este grupo es el que concentra el mayor porcentaje de hombres, lo que podría comportarse como un sesgo de confusión.

Por último, a pesar de que la comparación entre nuestros resultados con otros estudios internacionales pueden ser discutibles pues representan a muestras muy diferentes, con criterios de selección distintos; debemos señalar que la población estudiada presentó diámetros aórticos menores que los promedios obtenidos en el estudio de Lederle (Lederle et al., 1997), el estudio de mayor peso existente, en el que el promedio general de la muestra fue de $20 \mathrm{~mm}$, comparado con los $16,1 \mathrm{~mm}$ de nuestro estudio. Al desagrupar las muestras por sexo ocurre algo similar con 20 y $18 \mathrm{~mm}$ en los grupos de hombres y mujeres respectivamente para el estudio de Lederle; en comparación con los 17,9 y 14,9 de los grupos de nuestra población.

La novedad de la propuesta tiene que ver con que se trata de un estudio de corte transversal, con una muestra importante de sujetos, realizado en población nacional y de regiones; que nos orienta a que es posible que los DAP y DT de la AAI de la población blanco sean efectivamente menores a las reportadas a nivel internacional, lo que haría fundamental precisar aún más estos datos; dado que pudiesen generar un cambio en el punto de corte adoptado para definir la normalidad de estos diámetros, y por ende para plantear el diagnóstico de AAA.

Entre las limitaciones del estudio, se han de considerar potenciales sesgos tanto de medición (al haberse utilizado diferentes instrumentos de medición y habiéndose realizado las mediciones por dos observadores independientes), como de confusión con otras variables, como el sexo, que pudiesen haber interferido en los resultados (Manterola \& Otzen 2015).

A modo de conclusión, se puede señalar que el sexo masculino, la edad > 50 años; y una superficie corporal mayor; se asocian con DAP y DT de AAI más elevados. Y, que al parecer la población estudiada posee AAI de menor diámetro en promedio que lo descrito en la literatura internacional.

LAGOS, M. \& MANTEROLA, C. What are the normal ranges of the infrarenal aortic diameters measured with 2D ultrasound in subjects with no history of vascular disease? Int. J. Morphol., 34(3):1017-1023, 2016.

SUMMARY: The diameter of the abdominal aorta (AA), is a relevant factor to repair an abdominal aortic aneurysm. The aim of this study is to determine the normal range of the diameter of the infrarenal AA according 2D ultrasonography in patients with no history of vascular disease. Cross-sectional study, conducted in Hospital Regional of Temuco and Pitrufquen in 399 subjects over 15 years, with no history of vascular disease, who consulted for abdominal pain cadres were studied. 2D ultrasonography was performed with two different computers (General Electric RT3200 Advantage II and co Medison. Ltda. Model SA-600, both with $3.5 \mathrm{MHz}$ transducers.) and two independent observers. The outcome variables were anteroposterior diameters (DAP) and transverse (TD) of AA. Other variables of interest were: sex, age index weight / height and body surface. Descriptive statistics were applied, applying inferential $\mathrm{t}$ test for continuous variables, $\mathrm{x}^{2}$ for categorical variables and Kruskal Wallis ANOVA for comparison of groups; correlation studies and linear regression. DAP average was $16.1 \pm 2.2 \mathrm{~mm}(9-23 \mathrm{~mm})$ and DT average was $19.4 \pm 2.7 \mathrm{~mm}$ $(11-26 \mathrm{~mm})$. DAP and DT was significantly higher in the subgroup age $>50$ and in men. There was a positive correlation between weight / height-DAP ( $\mathrm{p}=0.0321)$ and index weight / height-DT $(\mathrm{p}=0.0052)$, the more relevant in the female subgroup index. Moreover, positive correlation between DAP and body surface area $(\mathrm{p}<0.0001)$ and DT and body surface area $(\mathrm{p}<0.0001)$ was demonstrated. Sex, age and body surface area are associated with higher DAP and DT. Apparently our population has lower average diameter aortas that described in the international literature.

KEY WORDS: Aorta; Abdominal Aorta; Abdominal aortic diameter; Aortic Aneurysm; Abdominal; Abdominal/ ultrasonography; ; Cross-Sectional Studies; 


\section{REFERENCIAS BIBLIOGRÁFICAS}

Allison, M. A.; Kwan, K.; DiTomasso, D.; Wright, C. M. \& Criqui, M. H. The epidemiology of abdominal aortic diameter. J. Vasc. Surg., 48(1):121-7, 2008.

Beales, L.; Wolstenhulme, S.; Evans, J. A.; West, R. \& Scott, D. J. Reproducibility of ultrasound measurement of the abdominal aorta. Br. J. Surg., 98(11):1517-25, 2011.

Bengtsson, H.; Sonesson, B. \& Bergqvist, D. Incidence and prevalence of abdominal aortic aneurysms, estimated by necropsy studies and population screening by ultrasound. Ann. N. Y. Acad. Sci., 800:1-24, 1996.

Bonnafy, T.; Lacroix, P.; Desormais, I.; Labrunie, A.; Marin, B.; Leclerc, A.; Oueslati, A.; Rollé, F.; Vignon, P. \& Aboyans, V. Reliability of the measurement of the abdominal aortic diameter by novice operators using a pocket-sized ultrasound system. Arch. Cardiovasc. Dis., 106(12):644-50, 2013.

Gürtelschmid, M.; Björck, M. \& Wanhainen, A. Comparison of three ultrasound methods of measuring the diameter of the abdominal aorta. Br. J. Surg., 101(6):633-6, 2014.

Länne, T.; Stale, H.; Bengtsson, H.; Gustafsson, D.; Bergqvist, D.; Sonesson, B.; Lecerof, H. \& Dahl, P. Noninvasive measurement of diameter changes in the distal abdominal aorta in man. Ultrasound Med. Biol., 18(5):451-7, 1992.

Lederle, F. A.; Johnson, G. R.; Wilson, S. E.; Chute, E. P.; Littooy, F. N.; Bandyk, D.; Krupski, W. C.; Barone, G. W.; Acher, C. W. \& Ballard, D. J. Prevalence and associations of abdominal aortic aneurysm detected through screening. Aneurysm Detection and Management (ADAM) Veterans Affairs Cooperative Study Group. Ann. Intern. Med., 126(6):441-9, 1997.

Liddington, M. I. \& Heather, B. P. The relationship between aortic diameter and body habitus. Eur. J. Vasc. Surg., 6(1):89-92, 1992.

Manterola, C. \& Astudillo, P. Checklist for reporting of descriptive observational studies. MINCIR initiative. Int. J. Morphol., 31(1):115-20, 2013.

Manterola, C. \& Otzen, T. Los sesgos en investigación clínica. Int. J. Morphol., 33(3):1156-64, 2015.

Peón, G. Aneurisma de la aorta abdominal. Disponible en: http:// www.drgerardopeon.com/pdf_enfer/enfer_4.pdf.Consultado el 6 de enero de 2016.

Poblete, R.; Draper, S.; Suárez, L.; Váquez, J.L.; Tevah, J.; Orrego, M. Morfología de la aorta abdominal en individuos presumiblemente sanos. Rev.Chil. Cir., 40:300-4, 1988.
Singh, K.; Bønaa, K.H.; Solberg, S.; Sørlie, D.G.; Bjørk, L. Intraand interobserver variability in ultrasound measurements of abdominal aortic diameter. The Tromsø Study. Eur. J. Vasc. Endovasc. Surg., 15(6):497-504, 1998.

Singh, K.; Bønaa, K.H.; Jacobsen, B.K.; Bjørk, L.; Solberg, S. Prevalence of and risk factors for abdominal aortic aneurysms in a population-based study : The Troms $\emptyset$ Study. Am. J. Epidemiol., 154(3):236-44, 2001.

Valdés, F.; Sepúlveda, N.; Krämer, A.; Mertens, R.; Bergoeing, M.; Mariné, L. et al. Frequency of abdominal aortic aneurysms in adult population with known risk factors. Rev. Med. Chil., 131:741-7, 2003a.

Valdés, F.; Bergoeing, M.; Krämer, A.; Mertens, R.; Canessa, R.; Lema, G. et al. Abdominal aortic aneurysms in patients over 80 years of age: conventional surgical treatment in 80 consecutive cases. Rev. Med. Chil., 131:981-6, 2003b.

Valdés, F.; Krämer, A.; Mertens, R.; Santini, A:, Canessa, R.; Lema, G. et al. Abdominal aortic aneurysm: course of morbimortality of elective surgery in 20 years. Rev. Med. Chil., 125:425-32, 1997.

Yucel, E.K.; Fillmore, D.J.; Knox, T.A.; Waltman, A.C. Sonographic measurement of abdominal aortic diameter: interobserver variability. J. Ultrasound Med., 10(12):681-3, 1991.

\author{
Correspondencia a: \\ Dr. Carlos Manterola \\ Departamento de Cirugía y CEMyQ \\ Universidad de La Frontera \\ Temuco \\ CHILE \\ E-mail: carlos.manterola@ufrontera.cl
}

Recibido : 16-04-2016

Aceptado: 26-07-2016 